\title{
Evidences for a double component in the emission of GRB 101023
}

\author{
A. V. Penacchioni $\left({ }^{1}\right)\left({ }^{3}\right)$, R. Ruffini $\left({ }^{1}\right)\left({ }^{2}\right)\left({ }^{3}\right), \operatorname{L} \cdot \operatorname{Izzo}\left({ }^{1}\right), \operatorname{M} \cdot \operatorname{Muccino}\left({ }^{1}\right)$, \\ C. L. Bianco $\left({ }^{1}\right)\left({ }^{2}\right)$, L. Caito $\left({ }^{1}\right)\left({ }^{2}\right)$ and B. Patricelli $\left({ }^{1}\right)$ \\ ${ }^{1}$ ) Dipartimento di Fisica, Sapienza Università di Roma and ICRA \\ Piazzale Aldo Moro 5, I-00185 Roma, Italy \\ (2) ICRANet - Piazzale della Repubblica 10, I-65122 Pescara, Italy \\ $\left(^{3}\right)$ Université de Nice Sophia Antipolis - Nice, Cedex 2, Grand Chateau Parc Valrose \\ Nice, France
}

ricevuto il 9 Marzo 2012

\begin{abstract}
Summary. - In this work we present the results of the analysis of GRB 101023 in the fireshell scenario. Its redshift is not known, so we attempted to infer it from the Amati Relation, obtaining $z=0.9$. Its light curve presents a double emission, which makes it very similar to the already studied GRB 090618. We called each part Episode 1 and Episode 2. We performed a time-resolved spectral analysis with RMFIT using different spectral models, and fitted the light curve with a numerical code integrating the fireshell equations of motion. We used Fermi GBM data to build the light curve, in particular the second NaI detector, in the range $(8.5-1000 \mathrm{keV})$. We considered different hypotheses regarding which part of the light curve could be the GRB and performed the analysis of all of them. We noticed a great variation of the temperature with time in the first episode, as well as almost no variation of the progenitor radius. We found that the first emission does not match the requirements for a GRB, while the second part perfectly agrees with being a canonical GRB, with a P-GRB lasting $4 \mathrm{~s}$.
\end{abstract}

PACS 97.60.Lf - Black holes.

PACS 98.70.Rz $-\gamma$-ray sources; $\gamma$-ray bursts.

\section{1. - Introduction}

GRB 101023 is a very peculiar source. It has been detected by many satellites, as Konus Wind, Swift and Fermi, and some on ground telescopes, as Gemini and GROND. Due to the lack of data, it has been impossible to determine its redshift. However, we tried to infer it using some empirical methods. What makes this source very interesting to study is the fact that it presents a double emission, being morphologically very similar to the already studied GRB 090618 [1]. We called these two parts Episode 1 and Episode 2, 
respectively. Episode 1 lasts $45 \mathrm{~s}$, while Episode 2 are the remaining $44 \mathrm{~s}$. The aim of this work is to compare and contrast these two sources, and from this to be able to determine all the parameters characterizing GRB 101023 (total energy, redshift, etc.). We performed a spectral analysis of both episodes and found that Episode 2 is well fit by a Band and a power-law model, while Episode 1 presents a thermal component that evolves in time following a broken power-law (for more details regarding the spectral analysis see [2]). We then performed a numerical simulation of the light curve and spectrum of Episode 2 with a numerical code called GRBsim, within the fireshell scenario [3-6]. We finally conclude that Episode 2 is a canonical GRB, while Episode 1 is the emission we see during the collapse of the progenitor core and the early phase of the formation of a black hole. We call this phenomenon a "proto-black hole".

In the fireshell scenario, the GRB emission originates from a process of vacuum polarization, resulting in pair creation in the so-called dyadosphere. In the process of gravitational collapse to a black hole [3] it is formed an $e^{ \pm}$plasma in thermal equilibrium, with total energy $E^{e^{ \pm}}$. The annihilation of these pairs occurs gradually and is confined in a shell, called "fireshell. This shell self-accelerates to relativistic velocities, engulfing the baryonic matter (of mass $M_{B}$, represented by the dimensionless parameter $B=M c^{2} / E^{e^{ \pm}}$) left over in the process of collapse and reaching a thermal equilibrium with it [4]. The fireshell continues to self-accelerate up to relativistic velocities [5] until it reaches the transparency condition. At this time we have a first flash of radiation, the Proper-GRB (P-GRB) [7]. The energy released in the P-GRB is a fraction of the initial energy of the dyadosphere $E^{e^{ \pm}}$. The residual plasma of leptons and baryons interacts with the circumburst medium as it expands, giving rise to a multi-wavelength emission: the extended afterglow. However, due to these collisions, the plasma starts to slow down. The structures observed in the prompt emission of a GRB are due to the inhomogeneities in this CBM, while in the standard fireball scenario [8] they are caused by internal shocks. In this way we need few parameters for a complete description of a GRB: the dyadosphere energy $E^{e^{ \pm}}$, the baryon load B and the CBM density distribution, $n_{C B M}$. In addition, we assume that there is spherical symmetry, and the energy released in the explosion $E_{i s o}$ is equal to the energy of the dyadosphere $E^{e^{ \pm}}$. From this approach, to sum up, the GRB bolometric light curve will be composed of two main parts: the P-GRB and the extended afterglow. Their relative energetics and their observed time separation are functions of the parameters $E^{e^{ \pm}}, B$ and $n$. We want to stress the fact that the emission of the P-GRB does not always coincide with what is called prompt emission in the fireball scenario. Indeed, within the fireshell model, this prompt emission corresponds to the gamma-ray emission, that addresses not only the P-GRB, but also the peak of the extended afterglow.

\section{2. - Observations}

GRB 101023 was detected by many instruments. One of them is Fermi GBM [9], which triggered on it with a trigger time of 309567006.726968 (in MET seconds). The burst was also detected by BAT [10] (see fig. 1), on board the Swift satellite [11], with a trigger time of 436981 (in MET seconds). Swift XRT [12] and Konus Wind [13] have also detected it, being the inferred location in complete agreement with the one determined by Fermi and Swift BAT. There have been also detections in the optical band by the Gemini telescope [14]. 


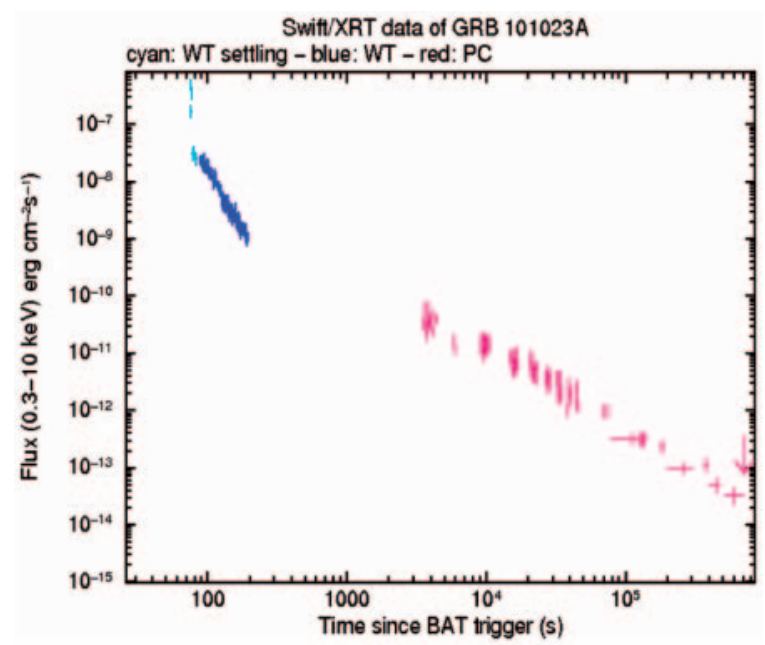

Fig. 1. - Count light curve of GRB 101023 obtained from Swift XRT detector.

\section{3. - Data reduction}

The Fermi GBM light curve, shown in fig. 2, is composed of two major pulses. The first one starts at the trigger time and lasts $45 \mathrm{~s}$. It consists of a small peak that lasts about $10 \mathrm{~s}$, followed by a higher emission that decays slowly with time. The duration, as well as the topology of this curve, lead to think that this may not be the case of a canonical GRB, but its origin may lie on another kind of source, which remains still unidentified [1]. The second pulse starts at $45 \mathrm{~s}$ after the trigger time and lasts $44 \mathrm{~s}$. It presents a peaky structure, composed by a short and weak peak at the beginning, followed by several bumps, big not only in magnitude but also in duration. This second emission, on the contrary, does have all the characteristics which describe a canonical GRB [2].

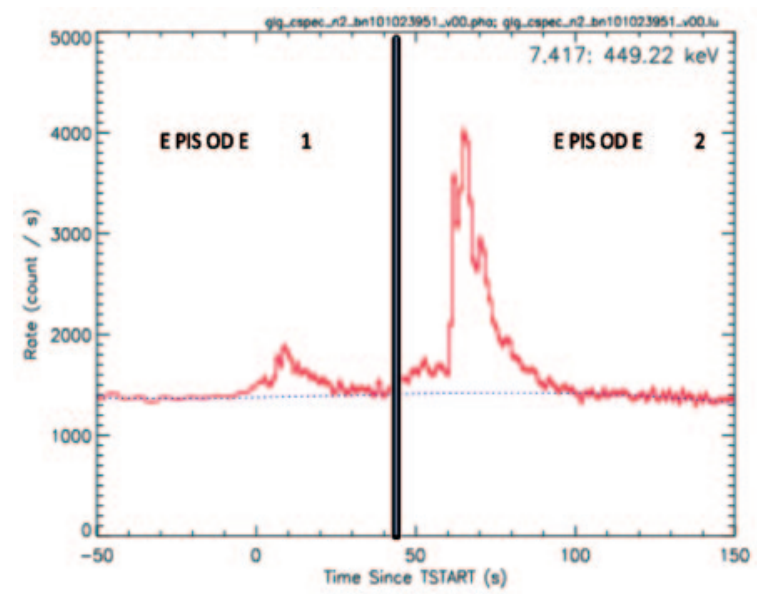

Fig. 2. - Count light curve of GRB 101023 obtained from Fermi GBM detector, with a bin time of $1 \mathrm{~s}$. The time is given with respect to the GBM trigger time of 22:50:04.73 UT, 23 October, 2010. The plot was obtained with the RMFIT program. The two-episode nature of the GRB is evidenced in analogy with GRB 090618. 


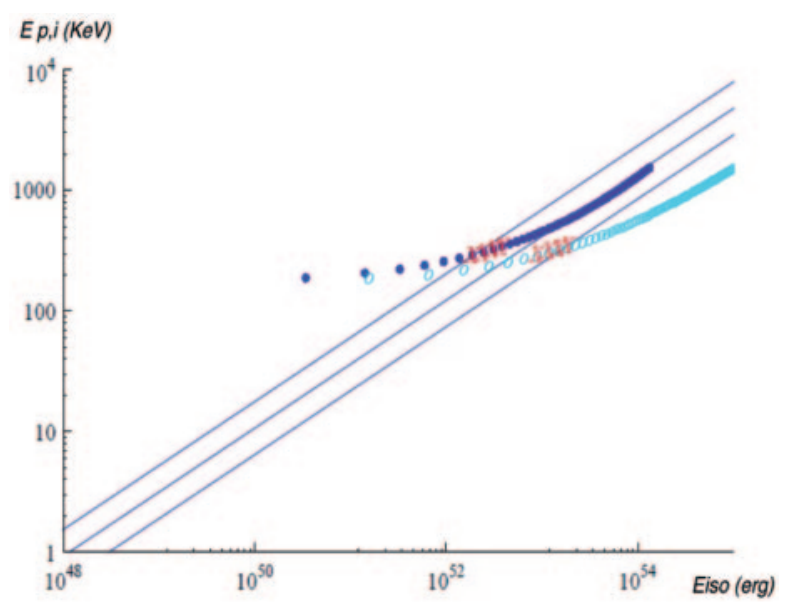

Fig. 3. - Plot of the relation between $E_{p, i}$ and $E_{i s o}$ for the second episode of GRB 101023, considering different values of the redshift. It can be seen that the plot lies within $1 \sigma$ for the range $z=0.3-1.0$.

\section{4. - Pseudo-redshift determination}

The redshift of this source is unknown, due to the lack of data in the optical band. However, we can infer a value of $z=0.9$ under the hypothesis that, as every long GRB, it should follow the Amati relation [15]. This relates the isotropic energy $E_{\text {iso }}$ emitted by a GRB to the peak energy in the rest frame $E_{p, i}$ of its $\nu F \nu$ electromagnetic spectrum. $E_{i s o}$ is the isotropic equivalent radiated energy, while $E_{p, i}$ is the photon energy at which the time averaged $\nu F \nu$ spectrum peaks. $E_{i s o}$ is given by

$$
E_{i s o}=\frac{4 \pi d_{l}^{2}}{(1+z)} S_{b o l},
$$

where $d_{l}^{2}$ is the luminosity distance, $z$ is the redshift and $S_{b o l}$ is the bolometric fluence (estimated between $1 \mathrm{keV}$ and $10 \mathrm{MeV}$ ), related to the observed fluence in a given detection band $\left(E_{\min }, E_{\max }\right)$ by

$$
S_{b o l}=S_{o b s} \frac{\int_{1 k e V /(1+z)}^{10 M e V /(1+z)} E \phi(E) \mathrm{d} E}{\int_{E^{\text {min }}}^{E^{\text {max }}} E \phi(E) \mathrm{d} E},
$$

with $\phi$ the spectral model considered for the spectral data fit. $E_{p, i}$ is related to the peak energy $E_{p}$ in the observer frame by

$$
E_{p, i}=E_{p}(1+z)
$$

We started our analysis under the hypothesis that Episode 2 is a long GRB. We computed the values of $E_{p, i}$ and $E_{i s o}$ for different given values of $z$ and plotted them in fig. 3. We found that the Amati relation is fulfilled by Episode 2 for $0.3<z<1.0$. This interval has been calculated at $1 \sigma$ from the best fit from the Amati relation. We chose $z=0.9$ because for this value of the redshift the energetics of this source are very similar to those of GRB 090618, whose redshift is known to be $z=0.54$. This is, the isotropic energy of events 1 and 2 in GRB 090618 are exactly the same as the isotropic energy of GRB1 and GRB2 in GRB 101023, respectively. 

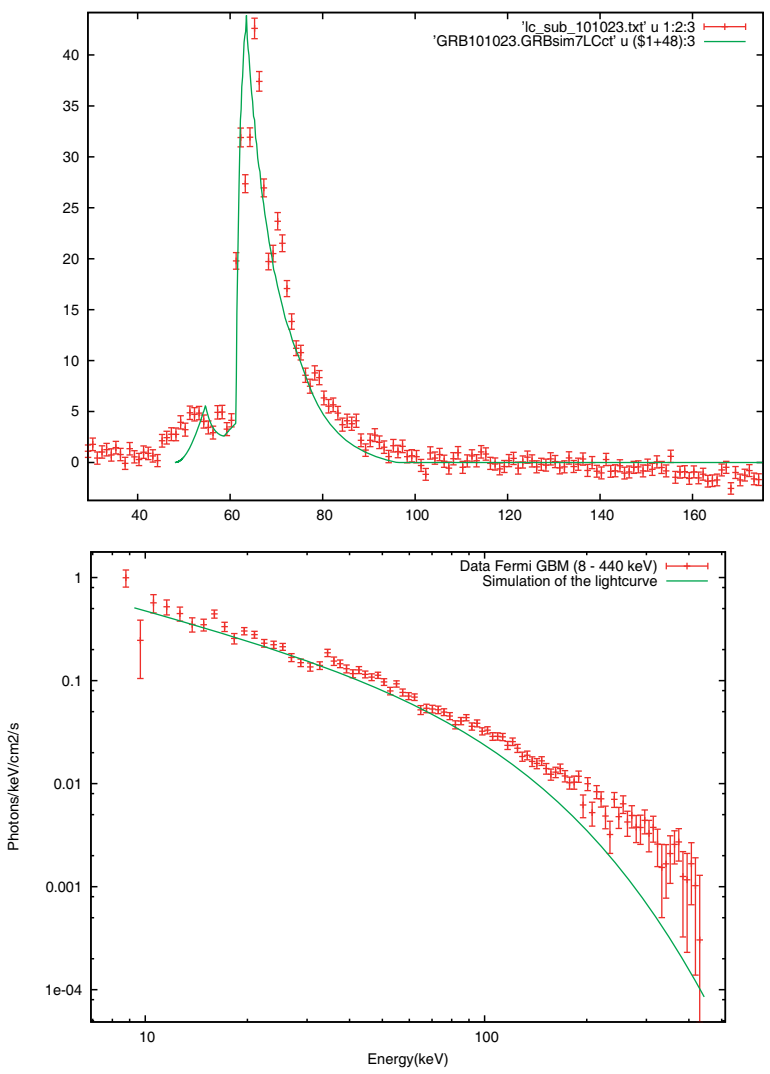

Fig. 4. - Top: Fit of the second major pulse of the light curve of GRB 101023. Bottom: Fit of the spectrum of Episode 2.

\section{5. - Data analysis and results}

To proceed with the fitting of the spectra, we defined first of all the time intervals we wanted to analyze making use of the gtbindef tool. We obtained a GTI file for the energy distribution. Secondly, we used gtbin to obtain the spectrum and in this way we are able to fit the data with different models, via XSPEC. We used a black body plus a power-law model and a band model. We can see that the second emission can be very well fitted by a black body plus a power-law model. In particular, we find for the P-GRB an observed temperature of $k T=13.5 \pm 2.8 \mathrm{keV}$, a photon index of $\gamma=2.5 \pm 0.7$ and a $\chi^{2}=0.91$. From these values we can infer a P-GRB energy $E_{P-G R B}=1.32 \times 10^{51} \mathrm{erg}$ (if we take the first $4 \mathrm{~s}$ of emission as the P-GRB) and a total energy of $E_{\text {iso }}=1.65 \times 10^{53} \mathrm{erg}$. We introduced these results in a numerical code to simulate the light curve (see fig. 4, left) and we found the following values for the parameters in the fireshell model: $B=4.8 \times 10^{-3}$, $k T_{t h}=20.83 \mathrm{keV}, \Gamma=206.81$, and a laboratory radius of $1.44 \times 10^{14} \mathrm{~cm}$. We also simulated the spectrum of Episode 2, shown in fig. 4, right. 

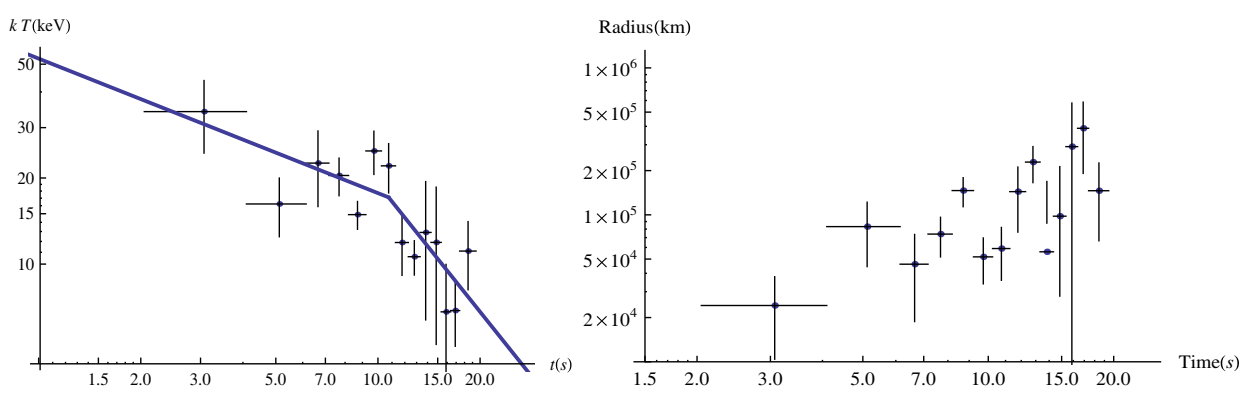

Fig. 5. - Left: Evolution of the observed temperature kT of the BB component. The blue line corresponds to a broken power-law fit. The indices of the first and second power-law are $\alpha=-0.47 \pm 0.34$ and $\beta=-1.48 \pm 1.13$, respectively. The break occurs at $11 \mathrm{~s}$ after the trigger time. Right: Evolution of the radius of the first episode progenitor.

5`1. Episode 1: Temperature and radius evolution. - To analyze Episode 1 more into detail, in order to identify the nature of this phenomenon, we plotted the temperature of the black body component as a function of time, for the first $20 \mathrm{~s}$ of emission (see fig. 5 , left). We note a strong evolution in the first $20 \mathrm{~s}$ of emission which can be reproduced by a broken power-law behavior, with $\alpha=-0.47 \pm 0.34$ and $\beta=-1.48 \pm 1.13$ being the indices of the first and second power-law, respectively [16]. We also plotted the radius of the most external shell with time (see fig. 5, right). Following Izzo et al. [1], the radius can be written as

$$
r_{e m}=\frac{\hat{R} D}{(1+z)^{2}}
$$

where $\hat{R}=\phi_{o b s} /\left(4 \pi \sigma T_{o b s}^{4}\right)$ is a parameter, $D$ is the luminosity distance, $\Gamma$ is the Lorentz factor and $\phi_{o b s}$ is the observed flux. We can see that the radius increases, but only slightly. From this it is possible to see that the plasma is expanding at non-relativistic velocities. According to the work of Arnett [17], there is an expansion phase of the boundary layers, while the iron core suffers a contraction. This is due to the presence of strong waves originated while the different shells of the progenitor mix during the collapse phase. This fact confirms the non-GRB nature for the first episode.

\section{6. - Conclusions}

As we can see from the results, the goodness of the spectral fits and the accordance between predicted and observed energies lead us to conclude that the second episode is indeed a canonical GRB, with a P-GRB that lasts $4 \mathrm{~s}$. On the contrary, the first episode does not present these characteristics. Within the fireshell scenario, we can conclude that we are in presence of some kind of mechanism that gives place to the formation of the black hole, when the core of the progenitor is collapsing. We call this a "proto-black hole". After this, it occurs the emission of the GRB as we already know it.

More details of this work will be presented in Penacchioni et al., 2011 (submitted). 


\section{REFERENCES}

[1] Izzo L., Ruffini R., Penacchioni A. V. et al., Astron. Astrophys., (2011) submitted.

[2] Penacchioni A., Ruffini R., Izzo L. et al., Astron. Astrophys., (2011) submitted.

[3] Ruffini R., Vereschagin G. and Xue S.-S., Phys. Rep., 487 (2010) 1.

[4] Ruffini R., Salmonson J. D., Wilson J. R. and Xue S.-S., Astron. Astrophys., 359 (2000) 855.

[5] Ruffini R., Astron. Astrophys., 138 (1999) 513.

[6] Ruffini R., Bianco C. L., Chardonnet P. et al., AIP Conf. Ser., 782 (2003) 16.

[7] Ruffini R., Bianco C. L., Fraschetti F., Xue S.-S. and Chardonnet P., Astrophys. $J ., 55$ (2001) L113.

[8] Meszaros P., Rep. Prog. Phys., 69 (2006) 2259.

[9] Page K. L. and Saxton C. J., GCN Circulars, 11368 (2010).

[10] Saxton C. J., Barthelmy S. D., Baumgartner W. H. et al., GCN Circulars, 11363 (2010)

[11] Gehrels N., Sarazin C. L., O'Brien P. T. et al., Nature, 437 (2005) 851.

[12] Burrows D., Hill J., Nousek J. et al., Space Sci. Rev., 120 (2005) 165.

[13] Golenetskit S., Aptekar R., Frederiks D. et al., GCN Circulars, 11384 (2010) .

[14] Levan A. J., Perley D. and D'Avanzo P., GCN Circulars, 11366 (2010) .

[15] Amati L., Mon. Not. R. Astron. Soc., 372 (2006) 233.

[16] Ryde F., Astrophys. J., 614 (2004) 827.

[17] Arnett W. D. and Meakin C., Astrophys. J., 733 (2011) 78. 